\title{
Importância da anamnese e do exame físico para o cuidado do enfermeiro
}

\author{
Importance of history and physical examination for the care of nurses \\ Importancia de la historia clínica y examen físico para el cuidado del enfermero
}

\author{
Neuma Santos', Patrícia Veiga', Renata Andrade' \\ 'Centro Universitário Jorge Amado. Salvador, BA
}

Submissão: 13/08/2009

Aprovação: $17 / 10 / 2010$

\section{RESUMO}

A anamnese e exame físico fazem parte da etapa do Histórico de Enfermagem do Processo de Enfermagem. Sua implementação visa o cuidado individualizado, holístico, humanizado e com embasamento científico. A revisão de literatura foi feita em bases indexadas como LILACS e SciELO, livros e revistas disponíveis nas bibliotecas locais publicadas entre os anos 2000 e 2009. O objetivo é descrever sobre a importância da anamnese e exame físico no cuidado prestado pelo enfermeiro. Os resultados desta pesQuisa possibilitarão Que graduandos de enfermagem e profissionais da área de saúde possam compreender melhor a importância da anamnese e exame físico para sua atuação profissional, aplicar devidamente todas as etapas da SAE e despertar o interesse por pesQuisas sobre esta temática.

Descritores: Anamnese; Exame físico; Cuidado, enfermagem.

\section{ABSTRACT}

The history and physical examination are part of data collection of the Nursing Process. Its implementation is aimed at individualized care, holistic, humane and scientific foundation. The literature review was carried out in indexed databases as LILACS and SciELO, books and journals available in local libraries as published between the years 2000 to 2009. The aim is to describe the importance of clinical history and physical examination in the care provided by nurses. The results of this research will enable nursing students and health professionals can better understand the importance of history taking and physical examination to their professional practice, implement properly all stages of NAS and arouse interest in research on this topic.

Key words: Medical history taking; Physical examination; Nursing care.

\section{RESUMEN}

La historia y el examen físico son parte del Historico de Enfermería del Proceso de Enfermería. Su aplicación está dirigida a la atención individualizada, integral, humana y fundamento científico. La revisión de la literatura se hizo sobre bases de datos indexados, LILACS y SciELO, libros y revistas disponibles en las bibliotecas locales, entre los años 2000 a 2009 . El objetivo es describir la importancia de la historia clínica y exploración física en el cuidado de las enfermeras. Los resultados de esta investigación permitirá a los estudiantes de enfermería y profesionales de la salud puedan comprender mejor la importancia de la anamnesis y exploración física para su práctica profesional, aplicar correctamente todas las fases del SAE y despertar el interés en la investigación sobre este tema.

Descriptores: Anamnesis; Examen físico; Atención de enfermería. 


\section{INTRODUÇÃO}

$\mathrm{Na}$ área da saúde, existe uma preocupação crescente dos diversos profissionais em aprimorar conhecimentos técnicos e científicos, estimulando assim seu desenvolvimento e aumentando suas responsabilidades, de forma Que o nível de assistência prestada ao cliente, família e comunidade seja Qualificado. Entre os enfermeiros, esta preocupação evidencia-se através do aprimoramento da aplicação da Sistematização da Assistência de Enfermagem (SAE) a Qual é composta pelas seguintes etapas: histórico de enfermagem Que compreende a anamnese e o exame físico; diagnóstico de enfermagem; prescrição de enfermagem,evolução de enfermagem e plano de alta. A anamnese e o exame físico, etapas deste processo, representam um instrumento de grande valia para a assistência, uma vez Que permite ao enfermeiro realizar o diagnóstico e planejar as ações de enfermagem, acompanhar e avaliar a evolução do paciente ${ }^{(1-2)}$. A partir do momento Que o enfermeiro é capaz de definir diagnósticos de enfermagem, estabelecer prescrições, avaliar a evolução do seu cliente ele estará apto para, juntamente com a equipe multiprofissional, planejar a alta do seu cliente. Para tanto, é muito importante Que sejam implementadas todas as etapas da SAE.

A SAE tem suas origens no processo de Enfermagem e a legislação brasileira ratifica isso através da Lei do Exercício Profissional, Lei $n^{\circ} 7498 / 86$, Que em seu artigo $8^{\circ}$, dispõe Que ao enfermeiro incumbe (...) a participação na elaboração, execução e avaliação dos planos assistenciais de saúde (...), ou seja, cabe a nós, enfermeiros, sistematizar, individualizar, administrar e assumir o papel de prestador do cuidado de enfermagem junto à equipe.

No Brasil, o emprego do processo de enfermagem foi incentivado por Wanda de Aguiar Horta, na década de 1970, em São Paulo, Que trouxe como referencial teórico a Teoria das Necessidades Humanas Básicas (NHB) de Maslow e Mohama. A Teoria das NHB engloba a Teoria da Motivação Humana de Maslow (1970) e de João Mohana (1964) Que classifica as necessidades humanas em psicobiológicas, psicosociais e psicoespirituais. Essas necessidades obedecem a uma hierarQuia, ou seja, níveis de valores a serem ultrapassados, onde, no momento em que o indivíduo realiza uma necessidade, surgem outras em seu lugar, exigindo sempre Que as pessoas busquem meios para satisfazê-las ${ }^{(3-4)}$. Ressaltamos que o Processo de Enfermagem é embasado numa metodologia científica, e contempla as seguintes etapas: levantamento de dados (histórico), diagnóstico, planejamento, execução e avaliação.

No entanto, com a aplicação da SAE o processo de cuidar em enfermagem torna-se mais amplo, através de planejamento ainda mais individualizado e holístico, mantendo o conhecimento científico como âncora deste cuidado. Para isso é imprescindível o levantamento sistematizado dos dados do paciente realizado no momento da internação ou na consulta de enfermagem Que consiste na primeira etapa da Sistematização da Assistência de enfermagem denominada de anamnese. Segundo Porto, 2001, Anamnese significa $A n a=$ trazer de volta, recordar mnese= memória, e é realizada através da técnica da entrevista.

A anamnese é definida como a primeira fase de um processo, na Qual a coleta destes dados permite ao profissional de saúde identificar problemas, determinar diagnósticos, planejar e implementar a sua assistência. Alguns autores apresentam Quatro tipos de dados coletados nessa primeira fase do Processo de Enfermagem Que são: dados subjetivos, objetivos, históricos e atuais. Estes podem ser obtidos, utilizando-se: a entrevista, a observação, o exame físico, os resultados de provas diagnósticas, a revisão de prontuário e a colaboração de outros profissionais ${ }^{(4)}$.

O Exame Físico, etapa relevante para o planejamento do cuidado do enfermeiro, busca avaliar o cliente através de sinais e sintomas, procurando por anormalidades Que podem sugerir problemas no processo de saúde e doença. Este exame deve ser realizado de maneira sistematizada, no sentido céfalo-caudal, através de uma avaliação minuciosa de todos os segmentos do corpo utilizando as técnicas propedêuticas: inspeção, palpação, percussão e ausculta. Para isto o enfermeiro necessita de recursos materiais, tais como esfigmomanômetro, estetoscópio, termômetro, diapasão, martelo de reflexo, espéculo de Collin, lanternas, otoscópios, luvas de procedimento estéril e não estéril, dentre outros. Além destes instrumentos básicos para a realização do exame físico, o enfermeiro deve utilizar os órgãos do sentido: visão, audição, tato e olfato para subsidiar o seu plano de cuidar/ cuidado.

Este estudo foi motivado pelo fato de Que durante as práticas acadêmicas evidenciou-se a ineficiente aplicabilidade da SAE, em especial, nas etapas Que correspondem a anamnese e o exame físico. Sabe-se Que todas as etapas da SAE devem ser implementadas tanto nos atendimentos domiciliares Quanto nas redes básicas e hospitalares.

O objetivo da pesquisa foi descrever sobre a importância da anamnese e do exame físico no cuidado prestado pelo enfermeiro. Assim sendo, este estudo é relevante porque possibilitará maior apreensão e socialização de conhecimentos sobre a temática, aumentar a aplicabilidade da SAE nos serviços e, consequentemente, motivar novas pesquisas sobre o assunto.

\section{MÉTODO}

Realizou-se uma revisão de literatura Que conduziu à compreensão do objeto de estudo e fundamentou a análise dos dados. Esta pesquisa foi desenvolvida através de material bibliográfico obtido de bases indexadas como LILACS e SciELO, entre os anos 2000 2009, além de livros e revistas disponíveis nas bibliotecas locais da cidade de Salvador, BA. Dessa forma, os temas consi-derados convergentes com os propósitos já explicitados são: anamnese, exame físico, cuidado, enfermagem.

\section{RESULTADOS E DISCUSSÃO}

A enfermagem é uma profissão secular Que desde os seus primórdios, teve sua formação voltada para o modelo de assistência centrado na execução de tarefas e procedimentos rápidos sob rígida disciplina. Os conhecimentos e atuação destes profissionais eram diretamente relacionados e subordinados aos saberes médico. Não haviam bases teóricas Que norteavam a profissão e, por isto esta categoria desenvolvia as suas atividades de modo, eminentemente, tecnicista. Em 1543, as primeiras Santas-Casas de Misericórdias foram fundadas, e, nesta época, a Enfermagem aí desempenhada, tinha cunho essencialmente prático. Em 1979, tem-se a criação do Centro de Estudos e Pesquisas em Enfermagem- CEPEN e com isso os currículos passam a ser centrados na assistência curativa e 
estágios realizados em hospitais. Assim sendo, sabe-se Que a formação do profissional enfermeiro enfatizava apenas as condutas práticas em detrimento do conhecimento científico e da correlação entre essa prática e a teoria.

No intuito de possibilitar Que a atuação de enfermagem tivesse um raciocínio mais crítico e clínico, surge o Processo de Enfermagem (PE) Que consiste em um instrumento metodológico empregado para favorecer o cuidado, além de organizar as condições necessárias para a sua ocorrência. Este era composto pelo diagnóstico, intervenções e resultados de enfermagem. O PE contribuía para o desenvolvimento de sistemas de classificação considerados instrumentos tecnológicos importantes para o processo de raciocínio e julgamento clínico dos elementos da prática profissional das enfermeiras ${ }^{(5)}$.

A metodologia do PE foi inserida nas escolas de enfermagem brasileiras, nos anos 1970, em São Paulo, a partir das contribuições de Wanda Horta Que trouxe como referencial teórico a Teoria das Necessidades Humanas Básicas de Maslow (1970) e Mahoma (1964). Organizado em três partes, o referido livro reuniu os escritos de Horta publicados anteriormente. Na primeira parte, Que trata da Filosofia da Enfermagem, a autora evidencia seu entendimento de que Filosofia é "pensar a realidade"; na segunda parte, ela descreve o Processo de Enfermagem; e, na terceira, apresenta o aplicativo do Processo de Enfermagem. Seus estudos, publicados em textos, artigos e livros Que têm como aspecto central o processo de enfermagem e a metodologia da assistência, foram utilizados em muitas Instituições de ensino de Enfermagem. Os escritos de Horta oportunizaram o desenvolvimento da enfermagem e a difusão das Teorias de Enfermagem ${ }^{(6)}$.

Em 25 de junho de 1986, a Lei $N^{\circ} 7.498$, normatizou o exercício profissional Quanto ao PE e estabeleceu como deveres privativos do enfermeiro o planejamento, a organização, a coordenação, a execução e a avaliação dos serviços, bem como a prescrição da assistência de enfermagem. O histórico de enfermagem Que compreende a anamnese e o exame físico ainda não estavam muito bem estabelecidos e implementados pela equipe de enfermagem. Coletar dados do cliente e examiná-lo ainda eram tidos como procedimentos e ou atuações médicas. Deste modo, como o enfermeiro podia prestar um conhecimento holístico, individualizado e mais humanizado ao seu paciente? Como definir diagnósticos de enfermagem, fazer prescrições e acompanhar a evolução dos seus pacientes?

Em 2002 o Conselho Federal de Enfermagem (COFEN), por meio da Resolução COFEN 272/2002, dispôs sobre a SAE. Assim, no seu artigo $3^{\circ}$, estabelecem Que deva fazer parte do prontuário do cliente/paciente/usuário, as seguintes etapas: Histórico de Enfermagem Diagnóstico de Enfermagem, Prescrição, Evolução e Relatório de Enfermagem ${ }^{(8-10)}$.

A SAE é uma abordagem sistematizada Que visa apoiar o trabalho da enfermeira, com a Qual ela organiza a assistência de enfermagem, planeja o seu fazer, executa cuidados e avalia a assistência prestada. A SAE é o modelo metodológico ideal para o enfermeiro aplicar seus conhecimentos técnico-científicos na prática assistencial.

É fundamental Que o enfermeiro assista e avalie o seu cliente numa visão holística no tocante aos aspectos biopsicossociais e espirituais. Quando o corpo e ou a mente sofrem, a pessoa é afe- tada em sua totalidade. Não se deve, portanto, enfocar apenas as partes que incomodam o individuo. Faz-se necessário que o enfermeiro tenha conhecimento técnico e científico para assistir o seu cliente, resgatando os princípios de necessidades humanas básicas citados por Básicas de Maslow , Mahoma e Horta. Ao realizar uma boa anamnese e um completo exame físico torna-se possível aumentar o elo de confiança entre profissional de saúde e cliente, coletar dados indispensáveis para o seu tratamento, identificar sinais e sintomas. Vale ressaltar que a anamnese e o exame físico, são as etapas inicias da SAE e as demais etapas (retirar; palavra repetida) Que compõem este método científico devem ser, rigorosamente, contempladas. Implantar todas as etapas da SAE no processo de cuidar é uma maneira de tornar a atuação da enfermagem mais científica, possibilitar melhores prognósticos e oferecer uma assistência de enfermagem de excelência.

O enfermeiro tem encontrado dificuldades na aplicação da metodologia da assistência de enfermagem como instrumento científico de trabalho. Tais dificuldades estão diretamente relacionadas às precárias condições de trabalho, ao Quantitativo insuficiente de funcionários e à formação acadêmica dos profissionais enfermeiros Que não esta voltada para a valorização da aplicabilidade da SAE e suas respectivas etapas. Ressalta-se Que as grades curriculares dos cursos de enfermagem têm contemplado este conteúdo, no entanto a oportunidade de correlacionar da teoria com a prática, no tocante a implementação da SAE, tem sido deficiente no cotidiano do enfermeiro. Outros aspectos relevantes à não aplicabilidade da SAE são o aprendizado tecnicista em detrimento de uma abordagem mais científica e a lógica da priorização do tratamento curativo. Vale ressaltar que é através da anamnese e do exame físico Que é possível conhecer melhor os nossos clientes e planejar uma assistência não só curativa, mas também preventiva.

A anamnese e o exame físico são partes integrantes do PE e consistem em um método sistematizado Que o enfermeiro utiliza para a prestação de cuidados humanizados ao cliente, ao mesmo tempo em que auxilia os demais profissionais a tomarem decisões e avaliarem os diagnósticos, prevenindo complicações e facilitando o tratamento do cliente $\mathrm{e}^{(11-12)}$. Aqui é realizada a coleta dos dados e a investigação feita nesta etapa é considerada de suma importância, pois se insuficiente ou incorreta pode levar ao diagnóstico de enfermagem errôneo e, conseQuentemente, resultará em um planejamento, implementação e avaliação inapropriados.

Etimologicamente, a palavra anamnese vem do grego anamnesis, e significa recordar. A anamnese, na prática clínica, consiste na rememoração dos eventos pregressos relacionados à saúde, na identificação dos sintomas e sinais atuais, com o intuito principal de fazer entender, com a maior precisão possível, a história da doença atual Que traz o paciente à consulta. A anamnese clínica, ao rememorar os acontecimentos referentes às condições de saúde, será tanto mais fidedigna Quanto mais for relatada pelo próprio paciente. Somente o paciente pode expressar suas próprias sensações. Exceções se fazem em condições de incapacidade deste, em urgências, ou Quando o paciente é ainda uma criança muito nova ${ }^{(8,11)}$.

A anamnese subsequente oferece um Quadro completo da história da doença atual e patológica pregressa da pessoa. Descreve o indivíduo como um todo e a forma como ele interage com o ambiente. É poder conhecer este paciente num primeiro e importante momento, o Que nos conduzirá de forma coerente as 
demais etapas desse contato.

O exame físico representa um instrumento de grande valia para a assistência, uma vez Que permite ao enfermeiro validar os achados da anamnese, identificar problemas, definir diagnóstico de enfermagem, planejar e implementar ações de enfermagem e acompanhar a evolução do paciente. Como etapa relevante, procura-se por anormalidades, sinais objetivos e verificáveis Que possam conter informações sobre os problemas de saúde significativos para a identificação dos diagnósticos de enfermagem, subsídios essenciais para o planejamento da assistência ${ }^{(12-13)}$.

A realização do exame físico de enfermagem é uma fase essencial da assistência sistematizada que deve ser executada de forma criteriosa pelos profissionais enfermeiros, visando uma atuação profissional científica. A identificação correta dos problemas apresentados pelos pacientes, através de uma avaliação clínica cuidadosa, torna-se fundamental para o desenvolvimento das ações do enfermeiro.

Assim, o Exame Físico é realizado de uma forma sistematizada, preferencialmente no sentido céfalo-plantar ou céfalo-caudal, com uma revisão minuciosa de todos os segmentos e regiões corporais. Para a realização do Exame Físico o examinador necessita de conhecimentos científicos em anatomia, fisiologia, fisiopatologia, diagnóstico por imagem, análises laboratoriais, patologia clínica e semiologia, sem os Quais ele não conseguirá detectar plenamente os problemas identificados e Que necessitam de sua intervenção ${ }^{(13)}$. Ao fazer um acompanhamento sistematizado de enfermagem devese valorizar não apenas o Que está sendo dito pelo cliente, mas também as suas linguagens não verbais

Ao implementarmos o método científico e sistematizado de cuidar dos nossos clientes onde todas as suas etapas possam ser contempladas, será possível prevenir, promover, proteger, recuperar e manter a saúde dos nossos clientes.

\section{CONCLUSÃO}

A enfermagem é uma profissão secular cuja atribuição era baseada em conhecimentos empíricos e eminentemente, tecnicistas. Ao longo dos anos a equipe de enfermagem, sob a supervisão dos enfermeiros, tem atuado de maneira cada vez mais científica. As bases do cientificismo na enfermagem do Brasil vieram do PE descrito e socializado por Wanda Horta. Recentemente, com o advento da SAE o cuidado prestado pela equipe de enfermagem tornouse mais específico e detalhado contemplando as seguintes etapas: Histórico de Enfermagem, Diagnóstico de enfermagem, Prescrição, Evolução e Relatório de enfermagem. Estes termos dão significado ao ato de registrar as etapas do PE e não substituem os termos usados para dar nome as etapas do PE.

É no histórico de enfermagem Que se aplica a anamnese e o exame físico. Através da anamnese e do exame físico é possível conhecer o cliente, estabelecer vínculos de confiança, identificar alterações biopsicossociais e espirituais e prosseguir definindo diagnósticos de enfermagem, traçando metas e ou prescrições de enfermagem, avaliando o paciente e realizando registros. O enfermeiro tem um papel fundamental na eQuação e resolução dos problemas apresentados pelos pacientes/clientes.

Atuando como mediador entre cliente, equipe multiprofissional, família e comunidade, o enfermeiro e a equipe de enfermagem auxiliam na resolutividade e enfrentamento dos problemas de saúde por eles diagnosticados. Assim sendo, é valioso que as instituições de ensino enfatizem esta temática na formação dos seus discentes, Que a Resolução COFEN 272/2002 Que discorre sobre SAE seja, efetivamente, aplicada pelos profissionais de enfermagem, porQue através desse método é possível realizar, além de outras etapas, uma boa anamnese, um completo exame físico, Que são itens de grande importância para a Qualidade dos serviços de enfermagem.

\section{REFERÊNCIAS}

I. Paula IC. A relevância do exame físico do idoso para a assistência de enfermagem hospitalar. Acta Paul Enferm 2005; I 8(3): $301-6$.

2. Sousa VD, Barros ALB. O ensino do exame físico em escolas de graduação em enfermagem do município de São Paulo. Rev Latino-am Enfermagem 1998; 6(3): 11-22.

3. Alves AR. Aplicação do Processo de Enfermagem: estudo de caso com uma puérpera. Rev Bras Enferm 2009; 60(3).

4. Cunha SMB, Barros ALBL. Análise da implementação da Sistematização da Assistência de Enfermagem, segundo o Modelo Conceitual de Horta. Rev Bras Enferm 2005; 58(5): 568-72.

5. Virginio NA, Nobrega MML. Validação de instrumento de coleta dedados de enfermagem para clientes adultos hospitalizados. Rev Bras Enferm 2004; 57(1): 53-6.

6. Boaventura AP. Ensino Do Processo de Enfermagem: percepção dos alunos do curso de graduação em enfermagem. XI Encontro Latino Americano de Iniciação Científica e VII Encontro Latino Americano de Pós-Graduação. 2007 fev 19; São José dos Campos (SP), Brasil. São José dos Campos: Universidade do Vale do Paraíba; 2009

7. Anselmi ML, Carvalho EC, Angerami ELS. Histórico de enfer-

magem: compreensão e utilização teórico-prática. Rev Esc Enferm USP 1998, 22(2): 18I-8.

8. Patine FS, Barboza DB, Pinto MH. Ensino do exame físico em uma escola de enfermagem. Are Ciênc Saúde 2004; I I (2).

9. Longaray VK, Almeida MA, Cezaro P. Processo de enfermagem: reflexões de auxiliares e técnicos. Texto Contexto Enferm 2008; 17(1): 150-7.

10. Kletemberg DF, Siqueira MD, Mantovani MF. História do Processo de Enfermagem na REBEn: 1960 - 1986. Esc Anna Nery Rev Enferm 2006; 10(3): 478-86.

11. Freitas EP, Nass F, Sponchiado F. Processo de enfermagem: Uma perspectiva para melhorar a Qualidade da assistência [monografia]. Florianópolis: Escola de Enfermagem da Universidade do Estado de Santa Catarina; 2008.

12. Barros ALB, Glashan RQ, Michel JML. Bases propedêuticas para a prática de enfermagem: uma necessidade atual. Acta Paul Enferm 1996; 9(1): 28-37.

13. Pires SB, Méier MJ. A sistematização do cuidado em enfermagem: uma análise da implementação [dissertação]. Curitiba: Programa de Pós-Graduação em Enfermagem, Universidade Federal do Paraná; 2007. 